\title{
Raviver une matière monumentale
}

Entretien avec Nicolas Michelin

\section{Christophe Didier et Nicolas Michelin}

\section{(2) OpenEdition}

\section{Journals}

Édition électronique

URL : https://journals.openedition.org/rbnu/3128

DOI : $10.4000 /$ rbnu. 3128

ISSN : 2679-6104

Éditeur

Bibliothèque nationale et universitaire de Strasbourg

Édition imprimée

Date de publication : 1 mai 2010

Pagination : 56-59

ISSN : 2109-2761

Référence électronique

Christophe Didier et Nicolas Michelin, « Raviver une matière monumentale », La Revue de la BNU [En ligne], 1 | 2010, mis en ligne le 01 janvier 2020, consulté le 12 juin 2021. URL : http:// journals.openedition.org/rbnu/3128; DOI : https://doi.org/10.4000/rbnu.3128

La Revue de la BNU est mise à disposition selon les termes de la Licence Creative Commons Attribution - Pas d'Utilisation Commerciale - Partage dans les Mêmes Conditions 4.0 International. 


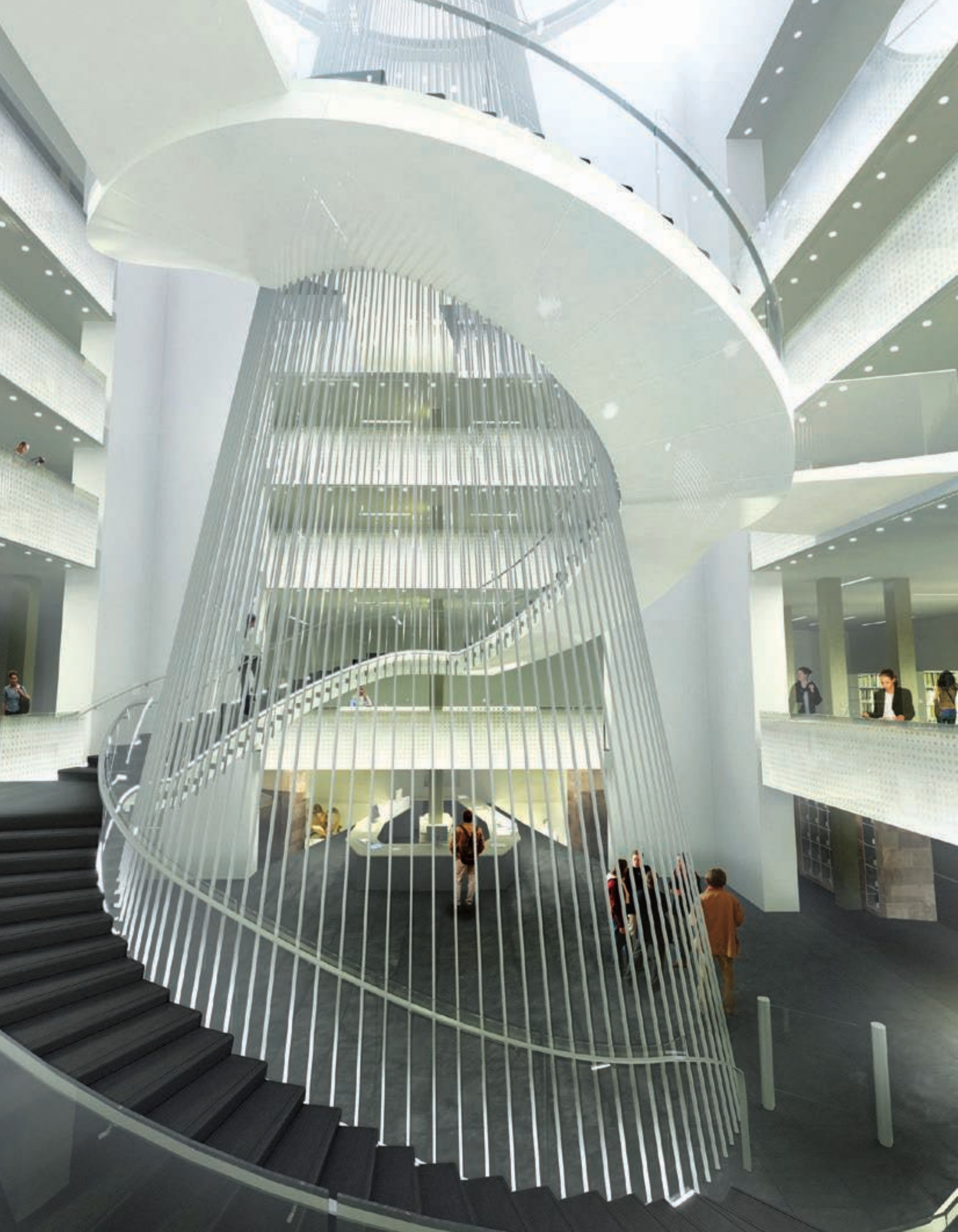




\title{
RAVIVER UNE MATIĖRE MONUMENTALE
}

\author{
Entretien avec Nicolas Michelin
}

L'agence de l'architecte et urbaniste Nicolas Michelin (ANMA), auteur entre autres de la réhabilitation en bâtiment universitaire de la Halle au farines à Paris et de plusieurs projets d'urbanisme (à Rennes, Metz, Nancy ou Mulhouse, pour n'en citer que quelques-uns), a été choisie en 2006 pour la restructuration du bâtiment principal

de la BNU. Ces travaux lourds qui contribueront à un complet changement de tous les espaces intérieurs vont naturellement avoir une incidence sur l'identité visuelle du lieu. Nous avons cherché à savoir comment Nicolas Michelin avait pensé renouveler celle-ci.

La BNU est un bâtiment marqué extérieurement par une esthétique datée, celle de l'historicisme de la fin du XIX ${ }^{\mathrm{e}}$ siècle. Comment cela a-t-il influencé votre approche du projet de restructuration?

N.M. Dans le bâtiment initial de 1895, l'extérieur reflète en quelque sorte l'intérieur, organisé de façon très symétrique et selon un plan centré. Cette esthétique qui guidait l'ordonnance de la bibliothèque allemande m'est apparue comme ce qui devait nous guider aussi dans le projet de restructuration. Celuici s'articule donc autour des idées de symétrie et de centre, et en ce sens, renoue avec le bâtiment d'origine qu'il me paraissait absurde d'ignorer, d'autant plus que le classement de l'enveloppe extérieure comme monument historique nous contraignait de toute façon. En ce sens, je tourne le dos au bâtiment des années cinquante, dont les restructurations avaient occulté en grande partie la force du plan cen- tral d'origine. En dégageant la coupole, je redonne à l'intérieur une partie de son lustre d'antan et rétablis en même temps sa logique structurelle. Donc, en ce sens, oui, le bâtiment d'origine a influencé mon approche, mais moins esthétiquement que structurellement. De ce point de vue, j’ai été moins sensible à la conservation des éléments des années cinquante (qu'on aurait pu aussi envisager, après tout) et j'ai plutôt choisi de retrouver la force structurelle des débuts.

Plus précisément, le message iconographique délivré en particulier par les médaillons et reliefs des façades vous a-t-il inspiré, gêné ou paru indifférent?

N.M. Il ne m’a ni gêné ni laissé indifférent ; l'extérieur reste de toute façon l'écrin de la bibliothèque. Protégé au titre des monuments historiques, il sera restauré comme tel et contribuera, demain comme 


\section{"A l'heure d'internet et des capacités virtuelles, les bibliothèques doivent montrer qu'elles ont elles aussi quelque chose d'exceptionnel à offrir dans les lieux biens réels qu'elles ouvrent au public..."}

aujourd'hui, de façon essentielle à l'essence du lieu. C'est aussi pourquoi nous l'intégrons, à notre façon, dans le projet. Le lecteur sera ainsi accompagné, en gravissant l'escalier principal, de deux murs vitrés de près de vingt mètres de long, sur lesquels le décor intérieur d'origine (au moins dans ses grandes lignes) sera visible au moyen d'images pixelisées placées à l'endroit où ce décor se trouvait jusqu'en 1950. Mais n'imaginez pas une reconstitution ! Ce sera bien plutôt la suggestion d'un décor, qu'on pourra voir ou ne pas voir - qui en tout cas ne s'imposera pas visuellement. On pourrait presque parler là de mémoire immatérielle, qui suggérera plus qu'elle ne montrera l'esthétique très décorée et très rythmée de l'époque. J'aime une certaine discrétion dans mes interventions, et en tout cas, j'ai une prédilection pour les décors qui ne s'imposent pas! Mais cette allusion à l'intérieur d'origine, dans un espace de transition et qui sera elle-même une transition imagée entre extérieur et intérieur, me semble importante dans le cas de la BNU. Il était de toute façon nécessaire d' " habiter " un peu cet espace, qui est assez long et constitue le premier contact de l'usager avec les lieux.

\section{A l'exception, donc, des images allusives de l'escalier d'entrée, votre projet se caractérise par une absence a priori d'éléments décoratifs au sens traditionnel du terme...}

N.M. Au sens traditionnel du terme, oui, si vous voulez, mais pas par une absence totale. L'élément phare du projet, le grand escalier central est aussi le lieu où va se matérialiser le second élément qu'on peut qualifier de décoratif, même si sa fonction va en fait au-delà. L'escalier central va se trouver sous la coupole. Or la particularité de la coupole sera que, contrairement à la façon dont l'éclairage se fait en pareil cas, le centre en sera opaque et la périphérie lumineuse. L'escalier sera, lui, soutenu en son centre par de grandes tiges d'acier inox qui permettront d'en suspendre les marches ; mais en même temps, la lumière rentrant par les côtés de la coupole sera diffusée vers le bas précisément grâce à ces tiges qui la réfléchiront. J'introduis là un élément à la fois structurant (par le soutien des marches) et décoratif (par la diffusion de la lumière) qui sera pour ainsi dire la signature de l'espace central, car avec les vitrages de l'escalier d'accueil, c'est le premier élément qu'on verra en pénétrant dans la bibliothèque. Le décor, tel que je le conçois, est surtout constitué de quelques grands éléments majeurs qui permettent de mettre en scène un effet spatial de l'architecture. En ce sens d'ailleurs, la salle de lecture principale, ouverte en encorbellement presque jusqu'au sommet du bâtiment (et recevant ainsi une lumière zénithale) en est un autre élément majeur.

\section{L'identité visuelle de la future BNU sera donc surtout marquée dans les espaces de circulation.}

N.M. La force décorative de l'escalier en spirale est pour moi primordiale, c'est vrai. Les espaces de travail seront plus fonctionnels et je n'ai pas envisagé pour eux de décors particuliers. Ce n'était d'ailleurs pas non plus le souhait des responsables de la bibliothèque...

\section{Comme architecte, vous prendrez part aussi aux décisions concernant la signalétique. Comment envisagez-vous celle-ci ?}

N.M. Pour moi, la signalétique doit faire partie de l'architecture, et ne doit pas forcément s'imposer comme un élément décoratif autonome. L'impression d'invasion de lettres, logos et pictogrammes qui peut vous saisir à l'entrée de certains bâtiments ne fait pas partie de mon esthétique. Je vois davantage la signalétique comme un prolongement de l'esprit du lieu, si l'on peut dire... Là encore, je plaide pour 
la discrétion. Bien sûr, une bonne signalétique doit se voir. Comment dire? Tout en gardant sa fonction, elle ne doit pas s'imposer. Je ne vous cache pas que ce n'est pas toujours évident à réaliser !

La BNU va vivre d'ici à 2014 sa troisième campagne de travaux depuis sa création. La seconde, celle des années cinquante, avait été marquée par une volonté de modestie dans son propos : on voulait s'éloigner du triomphalisme wilhelminien. Votre projet, qui réhabilite un plan centré, de grands espaces et un escalier monumental, signale-t-il l'époque d'une ambition retrouvée ?

N.M. Oui, incontestablement ! La BNU est un projet vraiment à part pour notre agence. On réhabilite beaucoup aujourd'hui, mais on n'a pas toujours l'occasion de travailler sur un bâtiment de cette envergure. En tout cas, j'assume l'ambition retrouvée ! A l'heure d'internet et des capacités virtuelles, les bibliothèques doivent montrer qu'elles ont elles aussi quelque chose d'exceptionnel à offrir dans les lieux biens réels qu'elles ouvrent au public, et à mon sens l'architecture est là pour les y aider. Moi qui reste souvent volontairement discret dans mes interventions (et qui resterai discret, comme je vous l'ai dit, quant au décor), je trouve que ce projet nécessitait la présence d'éléments bâtis remarquables et je revendique la restauration d'une certaine monumentalité : on va retrouver des choses qui étaient là (comme la coupole, les escaliers) mais avaient été dissimulés dans un aménagement intérieur moins lisible. Que voit-on de la coupole aujourd'hui une fois qu'on est entré dans la bibliothèque ? Mon projet la remet au centre des regards. J'aime assez la formule de l' " ordinaire extraordinaire ", que j'avais d'ailleurs employée dans un article ${ }^{1}$. Il me semble qu'elle s'applique assez à la restauration discrète d'une certaine monumentalité de la BNU...
On a souvent parlé, à propos des bâtiments très décorés des siècles précédents, de "message de pierre ". Cette notion de message se retrouve aussi dans plus d'un projet contemporain (la «transparence " de la chambre des députés allemands dans le nouveau bâtiment du Reichstag, le « retour à l'ethnique " du Centre culturel Tjibaou en Nouvelle Calédonie, pour ne prendre que ces deux exemples). Emploieriezvous une expression similaire à propos de votre travail ?

N.M. Oui, d'une certaine façon. Le message serait alors : on donne à un bâtiment une seconde chance, plutôt que de le démolir. C'est un peu la démarche qui m'avait guidé lors de la restructuration de la Halle aux farines pour l'université de Paris VII : le lieu conserve son identité première de bâtiment industriel, et en même temps il est aujourd'hui parfaitement admis comme lieu d'activité universitaire. Métamorphoser un monument, lui donner une autre vie (sans forcément le muséifier) est un concept important pour moi. Cela vaut d'ailleurs aussi dans des cas plus modestes comme ceux de l'habitat social. Il peut être à mon sens tout aussi valorisant (et pas forcément plus cher, contrairement à une idée reçue) de les remodeler plutôt que de les détruire pour reconstruire du neuf. Mais c'est là un autre sujet... En tout cas, il m'importe de redonner une matière monumentale à la BNU, et je pense qu'il y va aussi, par là, de sa future identité visuelle.

Propos recueillis par Christophe Didier

Note

1 - Paru dans la revue Archistorm, avril-mai 2009 